\title{
Positioning Control of Unmanned-plane based on Regional Feature Matching
}

\author{
Jianghong Li \\ School of Power and Energy, Northwestern Polytechnical University \\ Xi' an 710072 , China \\ Tel: 86-29-8846-0464Ｅ-mail: jhli@nwpu.edu.cn \\ Liang Chen \\ School of Power and Energy, Northwestern Polytechnical University \\ Xi'an, Shaan'xi 710072, China \\ Tel: 86-29-8846-0464Ｅ-mail: xbgydxxs@163.com \\ Yuanhu Cai \\ School of Power and Energy, Northwestern Polytechnical University \\ Xi'an 710072, China \\ Tel: 86-29-8846-0464Ｅ-mail: caiyh@nwpu.edu.cn
}

\begin{abstract}
Positioning control of UAV is one of the research areas of autonomous navigation. Regional feature matching algorithm is commonly used in the positioning control of UAVs. This paper proposes a regional feature matching algorithm, in which edges and gray-levels within image regions are extracted. The correspondences between regional features are then obtained in terms of invariant moments and chain-code representation of regions. The transformation parameters are estimated based on the centers of gravity of regions. Experimental results show the effectiveness of the proposed algorithm using salient regional features.
\end{abstract}

Keywords: UAV, Image matching, Edge, Regional features

\section{Introduction}

The aerial imaging system of UAV usually captures some salient regions (e.g., pool, square, farm field .etc). These regions have stable and prominent characteristics, and similar image features (e.g., grey-levels, textures, edges, etc.). In addition, they often have regular geometrical shape and the area of each region is often in a certain range. An image recorded by aerial imaging system of UAV usually contains a small number of regional features. The regional features and the relative positions between regions can be used for matching images in positioning control of UAV. In this paper, we propose a regional feature matching algorithm, in which the regional features are extracted at first (square, region center of gravity, shape, invariant rectangular) and then the centers of gravity of regions are utilized as control points to estimate the transformation parameters between images.

\section{Proposed Algorithm}

There are two major problems in regional feature matching, i.e., (1) how to reliably extract the salient regional features and describe them. The real physiognomy with much interference is very complex so that the regional feature extraction is not an easy task. (2) How to use the features to achieve accurate estimation. In order to extract regional features, regions are required to be segmented from the image using image segmentation technique. Here we segment the image into different regions according to unique means so that such regions have no intersection and each region has consistent gray-levels. The details of image segmentation are described as follows:

The image 
$\{\mathrm{f}(x, y) \mid 0 \leq x \leq \max (x), 0 \leq y \leq \max (y)\}$

is segmented into sub-regions $\mathrm{f}_{1}, \mathrm{f}_{2}, \mathrm{f}_{3} \cdots$ which satisfy the following requirements:

$\bigcup_{i=1}^{N} \mathrm{f}_{i}(x, y)=\mathrm{f}(x, y)$

namely, all the sub-regions constitute a whole image and $f_{i}$ is a connected region.

$\mathrm{f}_{i}(x, y) \cap \mathrm{f}_{j}(x, y)=\varnothing$

means that there is no intersection between two sub-regions. $f_{i}$ satisfies homogeneity requirement. The proposed algorithm combines the advantages of two segmentation methods, i.e., region growing and edge extraction, to achieve good image segmentation especially for complicated scene. Moreover, the proposed algorithm employs hierarchical search method based on image pyramid structure to improve the efficiency. The major steps of the proposed algorithm are shown in Figure 1. Specifically, the proposed algorithm first builds the pyramid structure, extracts the thick edges in the image, doing distance transform to edge image and choosing the obvious region, using the grey and edge as constraint to process region growth and getting region feature, calculating the region geometric feature and building the corresponding relation among region features, applying least square method to estimate the converted parameter in wide size which uses the region center of gravity as control point, choosing the sub-region with abundant features doing relevant matching to optimize parameter in thin size, at last getting the image change parameter.

\section{Regional feature extraction}

Image segmentation is not an easy task especially when the scene is quite complex. Single segmentation algorithm cannot gain satisfying segmentation result, and the combination of several segmentation algorithms is a key point of recent research. The region growing method is simple and robust, but it's a serial processing method. Therefore, its efficiency is low and the segmentation results are hard to control. Edge extraction is also a commonly used image segmentation method and consists of two main steps: edge detection and edge closing. Although edge extraction is effective, however, it's hard to find ideal close edges. This section describes the proposed image segmentation method that combines the advantages of region growing and edge extraction. The region growing mainly contains two parts: identification of seed point and growing rule. Usually, the seed point is identified by using some specific gray-level value, but it is hard to control the growing process. Because edge extraction can provide a rough segmentation result, it is able to control the region growing if region growing is confined to a finite region surrounded by edges. Using distance transform can choose the region with large area and highlight the important region feature. Furthermore, region growing is not only constrained by gray-level information, but also by edge characteristics. The segmentation process is summarized in Fig. 2.

Fig.3 shows an aerial image taken by UAV and Figure 4 depicts the edges extracted from the image shown in Fig.3.

Fig. 5 shows the result of image edge processed by distance transform, in which, the larger the gray value is, the larger the value of both the distance change and the specific area of the region. Fig.6 (a) demonstrates the candidate regions obtained from Fig.5 and Fig.6(b) shows the large regions in distance transform, which are formed by deleting the edges and the regions with small areas from Fig.6(a).

Fig.7(a) depicts the regions obtained by processing the candidate regions using region growing and rejecting the regions with small area after growing. Fig. 7(b) shows the boundaries of extracted regions.

After applying morphological processing to the image shown in Fig.7(a), the final result of extraction is shown in Fig.8.

\section{Estimation of Transformation parameters}

This section uses two real aerial images with known-parameters to test the effectiveness of the proposed algorithm. These two images cover similar scene with different resolutions. Fig.9 is a real-time image with size $172 * 190$, and Fig. 10 is the referenced image with size $224 * 242$.

The parameters selected in our experiments are: distance $\mathrm{L}=3$, region area $\mathrm{S}>30$, region growth $\mu=2$, distance constraint $d_{t=1}$. The above parameters are empirically chosen based on several experiments. Doing matching calculation to the extracted region features (the parameters applied in feature matching are: threshold of combined moment $T_{d}=0.07$, threshold of chain-code relevance $T_{c}$ is 0.8 ) gets 7 corresponding regions, Table 1 shows the coordinates of the center of gravity of regions. The estimates of transformation parameters are: rotation factor is 21.0414 degree and proportion factor is 1.3444 . The transformation equation of real-time image to referenced image is: 
$\left\{\begin{array}{l}x^{\prime}=47.9559+1.2548 x-0.4827 y \\ y^{\prime}=-44.3442+0.4827 x+1.2548 y\end{array}\right.$

RMS-Root Mean-Square Errors is a commonly used judge standard in matching accuracy, which is defined as:

$\operatorname{RMS}=\sqrt{\frac{\sum_{i=1}^{N}\left[\left(x^{\prime}-x\right)^{2}+\left(y^{\prime}-y^{2}\right)\right]}{N}}$

$\left\{\begin{array}{l}x^{\prime}=t_{x}+x \llbracket s \cos \theta-y \llbracket s \sin \theta \\ y^{\prime}=t_{y}+x \llbracket s \sin \theta+y \llbracket s \cos \theta\end{array}\right.$

In this paper, we use RMS to measure the matching accuracy. Table 2 lists the matching errors of the centers of gravity of corresponding regions. It is seen from Table 2 that the average matching error of all the 7 control points is 1.2883 , the maximum error of a single point is 1.9206 , which proves the matching algorithm has excellent matching accuracy.

\section{Conclusions}

This paper proposes a image matching algorithm based on region features for positioning control of UAV and its accuracy has been proved by experiment. Furthermore, a regional features extraction method using edge and image grey information as constrain is proposed which applies region growing and extracts the region with comparatively large area. Experiments have proved the algorithm can be used in solving the extraction of regional features of complicated scene.

\section{References}

Goshtasby, G. C. Stockman and C. V. Page. (1986). A region-based approach to digital image registration with subpixel accuracy. IEEE Trans. on Geoscience and Remote Sensing, 1986, 24(3): 390-399.

Hui Li, B. S. Manjunath and S. K. Mitra. (1995). A contour-based approach to multisensor image registration. IEEE Trans. on Image Processing, 1995, 4(3): 320-334.

J.P. Fan, D.K.Y. Yau, A.K. (2001). Elmagarmid and W.G. Aref. Automatic image segmentation by integrating color-edge extraction and seeded region growing. IEEE Trans. on Image Processing, 2001, 10: 1454-1466.

R. Adams and L. Bischof. (1994). Seeded region growing. IEEE Trans. on PAMI, 1994, 16: 641-647.

S. Dinggang and H. S. Horace. (1999). Discriminative Wavelet Shape Descriptors for Recognition of 2-D Patterns. Pattern Recognition 1999, 32: 151-165.

S.A. Hojjatoleslami and J. Kittler. (1998). Region growing: a new approach. IEEE Trans. on Image Processing, 1998, 7(7): 1079-1084.

Xiaolong Dai and S. K. Khorram. (1999). A teature-based image registration algorithm using improved chain-code representation combined with invariant moments. IEEE Trans. on Geoscience and Remote Sensing, 1999, 37(5): 2351-2362.

Zhang yujin. (2000). Graph engineering (above): graph process and analysis. Beijing: Tsinghua University Press, 2000.

Zhong kongde. (1996). Signal processing. Beijing: Tsinghua University Press. 
Table 1. corresponding control point

\begin{tabular}{|l|l|l|l|l|}
\hline \multirow{2}{*}{ Control point pair } & \multicolumn{2}{l|}{ Real-time image } & \multicolumn{2}{l|}{ Referenced image } \\
\cline { 2 - 5 } & $x$ & $y$ & $x^{\prime}$ & $y^{\prime}$ \\
\hline 1 & & & & 202.2111 \\
\hline 2 & 74.6599 & 169.3019 & 59.2050 & 162.7242 \\
\hline 3 & 56.3765 & 143.1000 & 50.0167 & 141.6800 \\
\hline 4 & 57.1597 & 126.4958 & 58.7467 & 109.5950 \\
\hline 5 & 65.1743 & 98.3527 & 81.4457 & 220.5187 \\
\hline 6 & 106.2676 & 171.5282 & 99.1567 & 53.9287 \\
\hline 7 & 72.2512 & 50.0171 & 113.0793 & 110.2338 \\
\hline
\end{tabular}

Table 2. RMS error of control point

\begin{tabular}{|l|l|l|l|l|l|l|l|}
\hline $\begin{array}{l}\text { Control } \\
\text { point }\end{array}$ & 1 & 2 & 3 & 4 & 5 & 6 & 7 \\
\hline $\mathrm{X}$ & 0.7097 & 0.3959 & 0.1281 & 0.8143 & 0.6558 & 1.3926 & 0.0742 \\
\hline $\mathrm{Y}$ & 1.9206 & 0.2957 & 0.2919 & 0.9321 & 1.6638 & 0.6363 & 0.0291 \\
\hline RMS & 1.2883 & \multicolumn{7}{|l|}{} \\
\hline
\end{tabular}

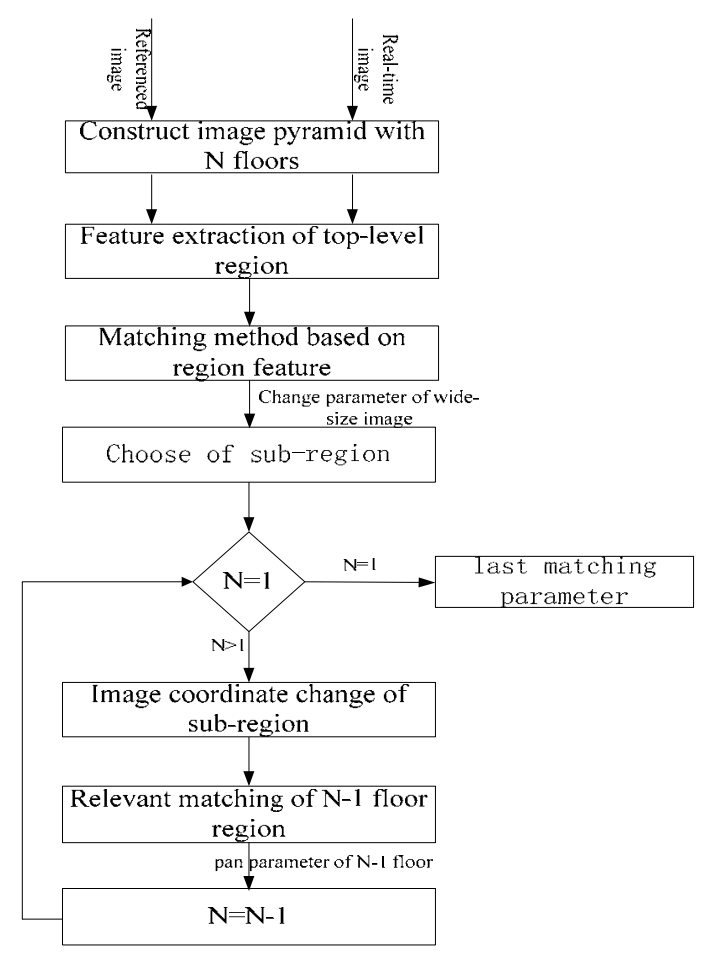

Figure 1. algorithm processing 


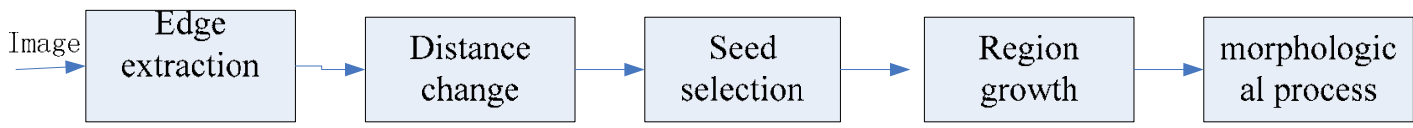

Figure 2. extract process of region characteristics

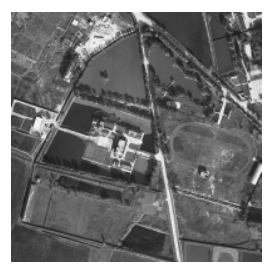

Figure 3. aerial photography

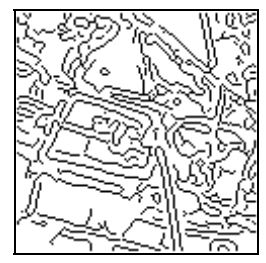

Figure 4. result of edge extraction

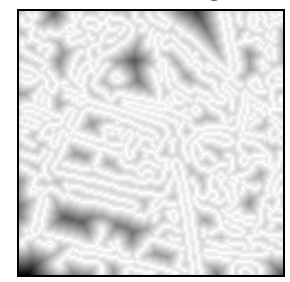

Figure 5. result of distance change

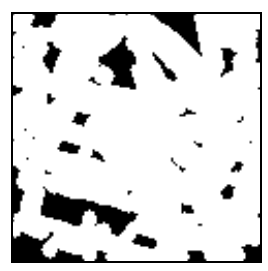

(a)

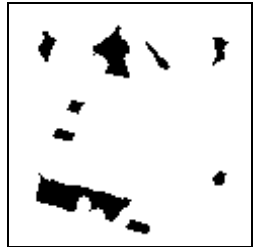

(b)

Figure 6. extraction of candidate region

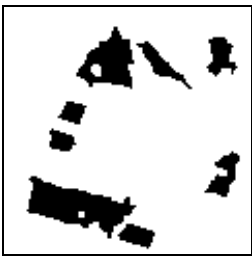

(a)

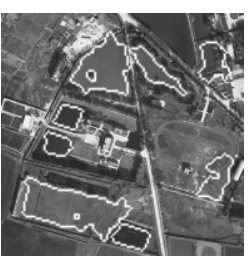

(b)

Figure 7. extraction results of region characteristics

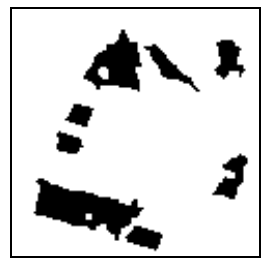

Figure 8. ultimate extraction results 


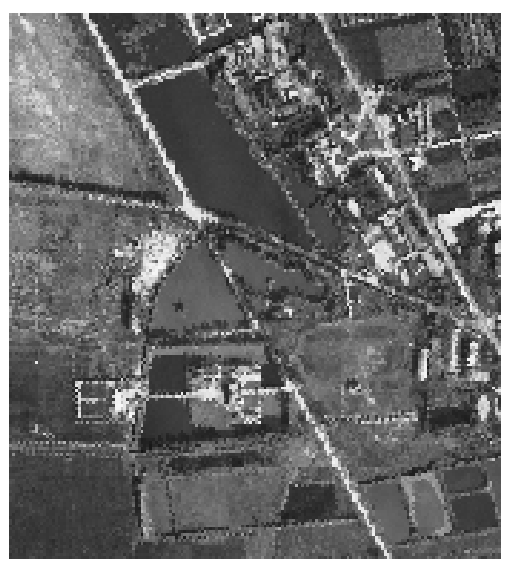

Figure 9. real-time image

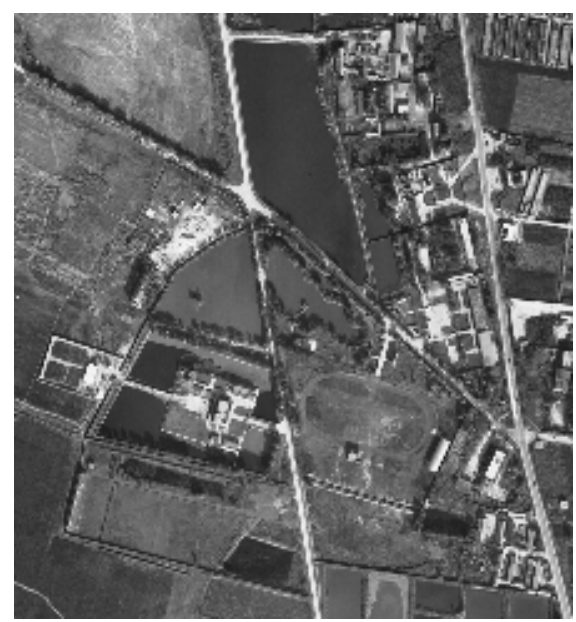

Figure 10. referenced image 